\title{
Power Spectral Analysis of Heart Rate Variability in a Horse with Atrial Fibrillation
}

\author{
Masayoshi KUWAHARA, Atsushi HIRAGA ${ }^{1)}$, Tsurayuki NISHIMURA ${ }^{2}$, Hirokazu TSUBONE and Shigeru SUGANO \\ Department of Comparative Pathophysiology, Graduate School of Agriculture and Life Sciences, The University of Tokyo, 1-1-1 Yayoi, \\ Bunkyo-ku, Tokyo 113, ${ }^{1)}$ Equine Research Institute, Japan Racing Association, 321-4 Togami-cho, Utsunomiya-shi, Tochigi 320, and \\ ${ }^{2}{ }^{2} H i d a k a$ Nosai, 2-5-5 Urakawa-cho Sakaimachi Higashi, Urakawa-gun, Hokkaido 057, Japan \\ (Received 13 June 1997/Accepted 19 August 1997)
}

ABSTRACT. This study has demonstrated the power spectral analysis of heart rate variability in a horse with atrial fibrillation. A large peak in the high frequency (HF) area of the power spectrum appeared in the horse. Hourly heart rate, the low frequency (LF) power, the HF power, and LF/HF ratio were almost constant during the recording period. The values of HF and LF power in the horse with atrial fibrillation were much larger than those in normal horses. The normalized unit of HF (HF n.u.) was much larger than that of LF (LF n.u.). Furthermore, the LF/HF ratio was very small in the horse. These results suggest that the ventricular rhythm has a respiratory related periodicity in the horse with atrial fibrillation and the predominant parasympathetic activity may modulate the intrinsic behavior of the atrioventricular node during atrial fibrillation. — KEY WORDS: atrial fibrillation, equine, heart rate variability.

Heart rate variability has provided increasing interest as a non-invasive index of autonomic nervous function [1, 13]. We have recently utilized power spectral analysis of heart rate variability in some animal species including horses to characterize quantitatively autonomic nervous system regulation of cardiovascular activity $[7,8,10]$. The power spectrum of heart rate variability in the horse resembled power spectra derived from humans, rats and dogs. Two major spectral components were observed. The high frequency (HF) component was clearly reflected on the respiratory frequency. The low frequency (LF) component corresponded to fluctuations of vasomotor and baroreceptor. The HF power is generally thought to reflect primarily parasympathetic nervous function, while the LF power reflects both the sympathetic and parasympathetic nervous functions. Further, the LF/HF ratio is considered as an index of the cardiac sympathovagal balance.

The equine heart exhibits a variety of different rhythms. Because it is thought that these heart rate variations are associated with fluctuations in the tone of the autonomic nervous system [15], it would be important to investigate the underlying autonomic nervous function of animals with arrhythmias. Atrial fibrillation is the most common arrhythmia affecting performance in horses [2, 4, 5, 12]. Meijler et al. [11] have shown that the periodicity of the ventricular rhythm is present in the horse with atrial fibrillation, but the rhythm is random in the dog and human. Although the autonomic nervous system might alter the ventricular response to atrial fibrillation, there was no direct evidence for the functional modulation of autonomic nervous system underling atrial fibrillation in the horse. We therefore applied the power spectral analysis of heart rate variability to estimate the role of the autonomic nervous system and the modulation of the intrinsic behavior of the atrioventricular (AV) node during atrial fibrillation.

The horse, "Shinzan", has been a legendary racehorse in Japan. Because a persistent type of atrial fibrillation was observed in the horse as reported previously [9], Holter ECG recordings were made with a two-channel recorder
(Fukuda Denshi Co., Ltd., Tokyo, Japan). The ECG was recorded in the stall from 16:00 to 6:00 using the ApexBase lead. An analysis was performed on an ECG processor analyzing system (Softron Co., Ltd., Tokyo, Japan) for power spectral analysis, as described previously [8]. Briefly, the computer program first detected $\mathrm{R}$ waves and calculated the R-R interval tachogram as the raw ventricular rate variability in sequence order. From this tachogram, data sets of 512 points were resampled at $2.5 \mathrm{~Hz}$. We then applied each set of data to the Hamming window and the fast Fourier transform to obtain the power spectrum of the fluctuation. Squared magnitudes and the products of the computed discrete Fourier transforms were averaged to obtain spectral estimates. A one-hour spectral plot was prepared from the average of 90 spectra computed over 3min periods. We obtained heart rate, the LF power, the HF power, and the $\mathrm{LF} / \mathrm{HF}$ ratio from recording. We set $\mathrm{LF}$ at $0.01-0.07 \mathrm{~Hz}$ and $\mathrm{HF}$ at $0.07-0.6 \mathrm{~Hz}$ according to the previous study [8]. The LF and HF components of the power spectrum were evaluated in both absolute values and normalized units (n.u.) [14]. Results were expressed as the mean \pm S.D.

The ECG showed a typical pattern of atrial fibrillation with the ventricular rate averaging 48 beats/min over $14 \mathrm{hr}$ (Fig. 1). Very coarse $f$ waves existed in the ECG. A tachogram and a histogram of RR intervals and a power spectrum of heart rate variability from the horse with atrial fibrillation are shown in Fig. 2. The longest RR intervals have a duration of $4,120 \mathrm{msec}$. The median RR interval is $1,050 \mathrm{msec}$. A large peak in the HF area of the power spectrum appeared in the horse. Because the HF peak corresponds to respiratory frequency, this result shows that the ventricular rhythm has a periodicity linked to respiration in the horse with atrial fibrillation. The values of HF $\left(145,078 \pm 18,383 \mathrm{msec}^{2}\right)$ and LF $\left(24,585 \pm 4,633 \mathrm{msec}^{2}\right)$ power in the horse with atrial fibrillation were much larger than that in the normal horses as described in previous study [8]. The LF n.u. and HF n.u. were $14.5 \pm 2.1$ and $85.5 \pm$ 2.1 , respectively. Furthermore, the $\mathrm{LF} / \mathrm{HF}$ ratio $(0.17 \pm 0.03)$ 
(A)

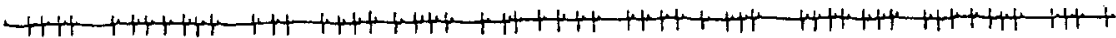

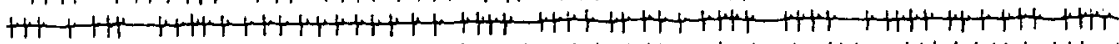

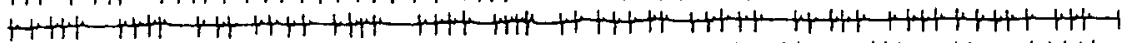

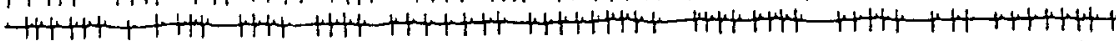

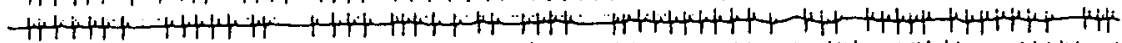

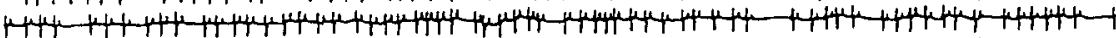

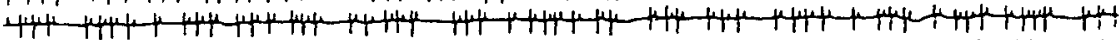

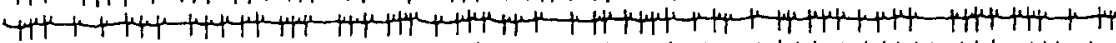

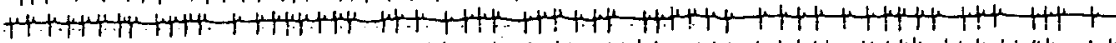

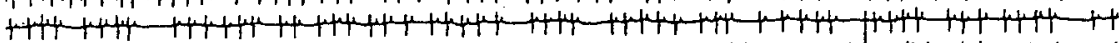

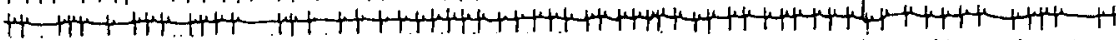

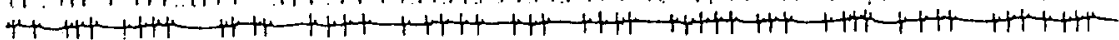

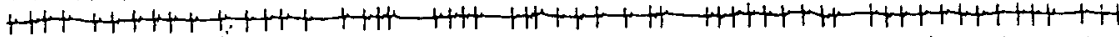

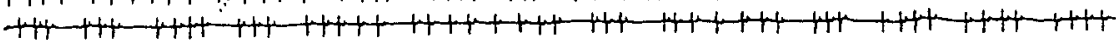

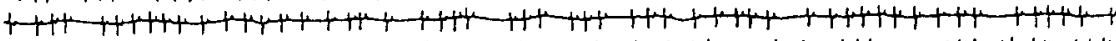

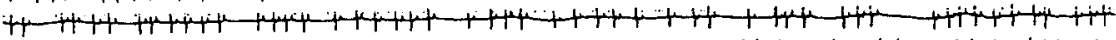

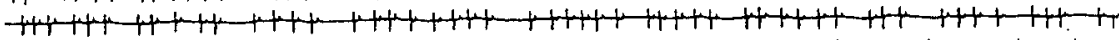

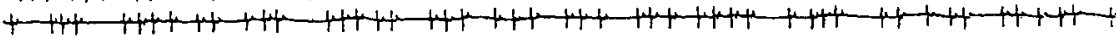

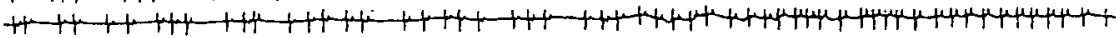
.

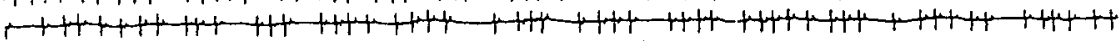

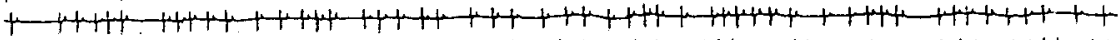

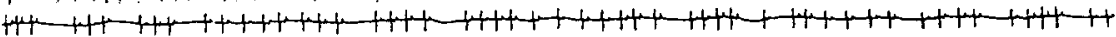

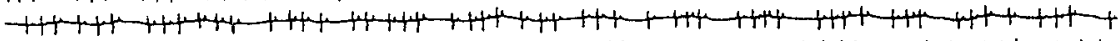

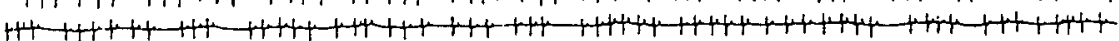

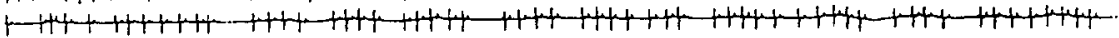

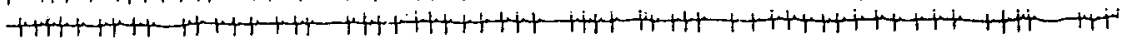

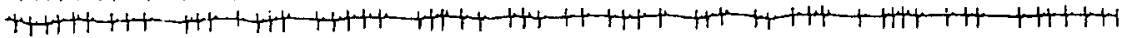

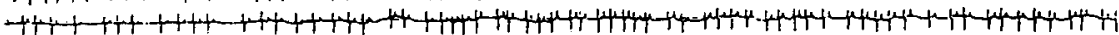

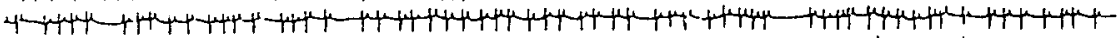

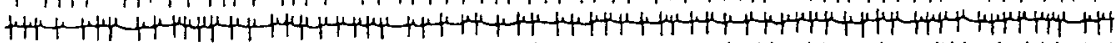

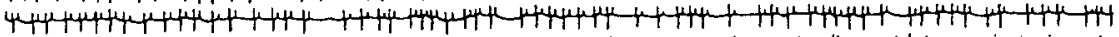

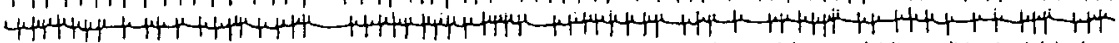

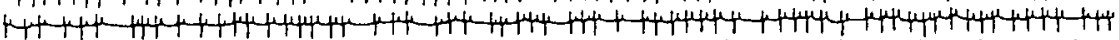

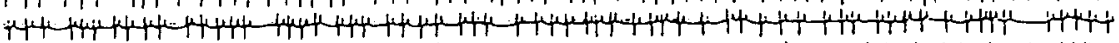

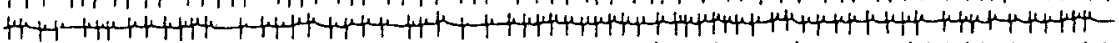

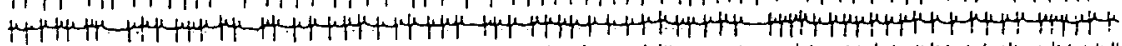

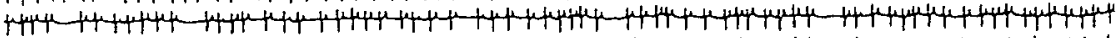


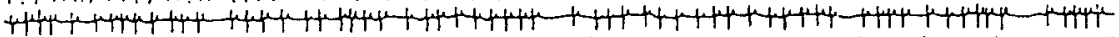

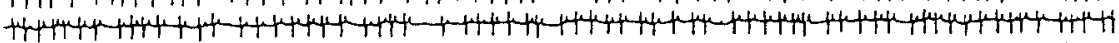

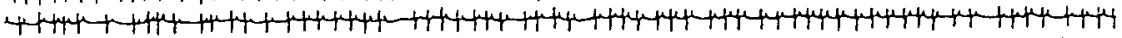

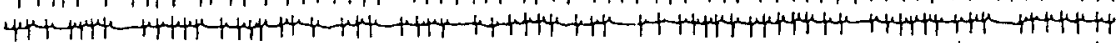

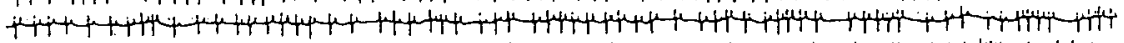

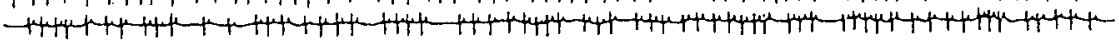

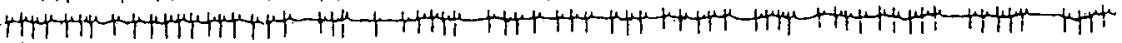

(B) $\sqrt{5 \mathrm{sec}}$

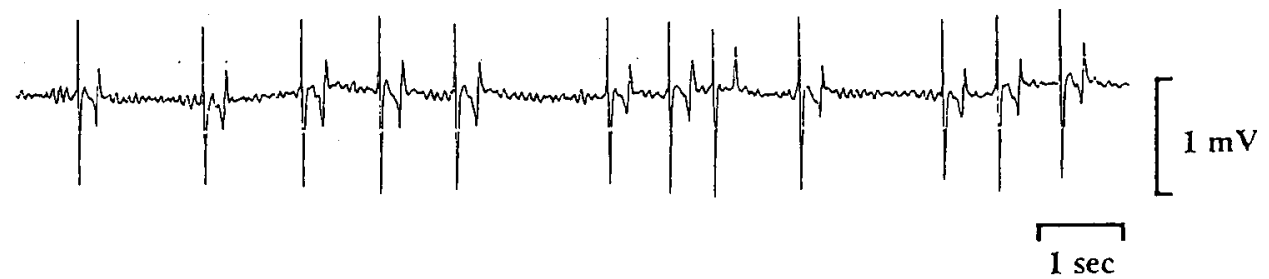

Fig. 1. ECG recording from Apex-Base Lead by a Holter ECG recorder showing the atrial fibrillation. (A) Compressed ECG for $1 \mathrm{hr}$ (B) Extended ECG waves. Very coarse f waves existed in the ECG.

in the horse was very smaller than that in the normal horses [8]. Hourly heart rate, the HF and LF power, and the LF/
HF ratio were almost constant during the recording period. These results suggest that the ventricular rhythm has a 
(A)
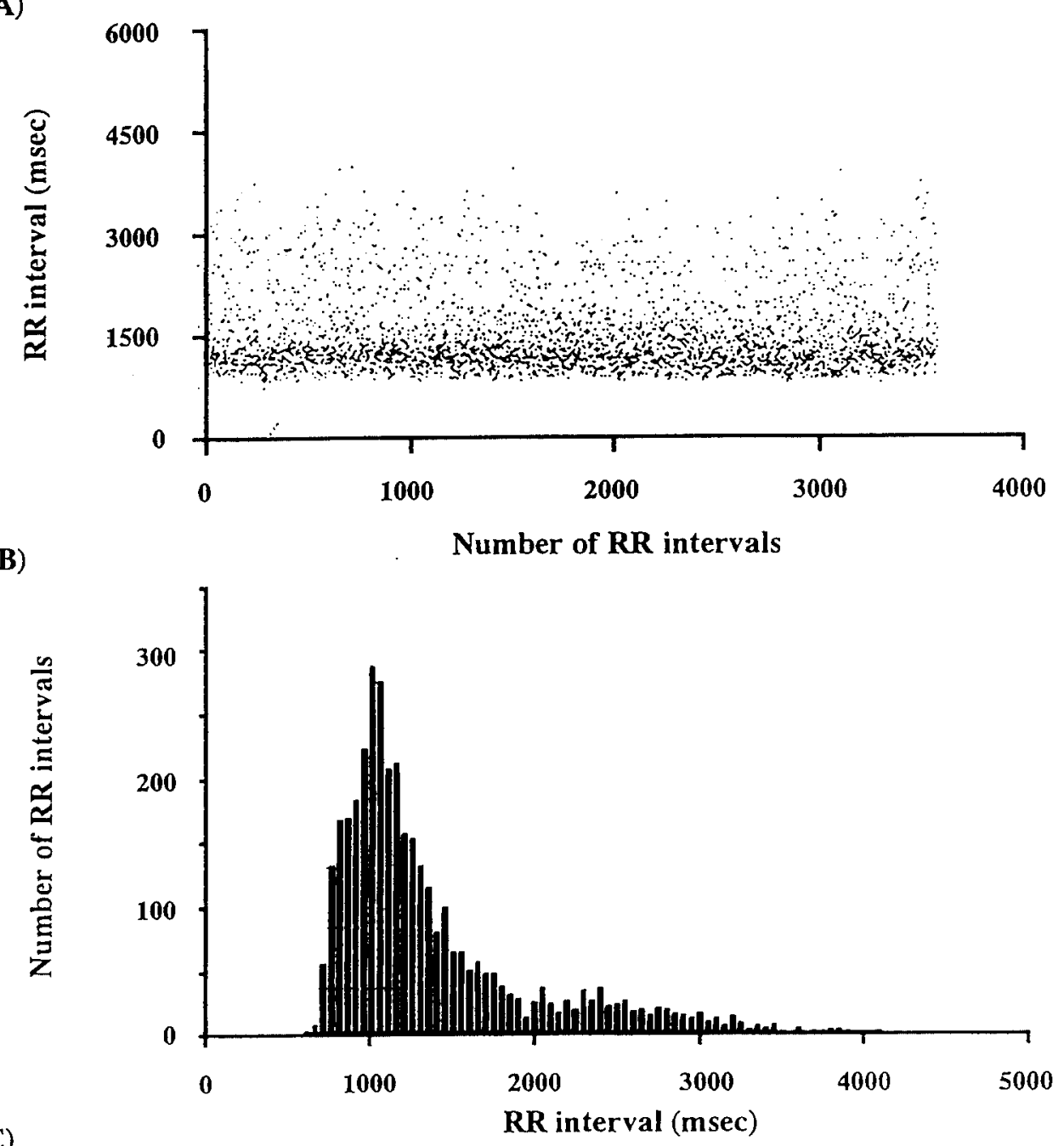

(C)

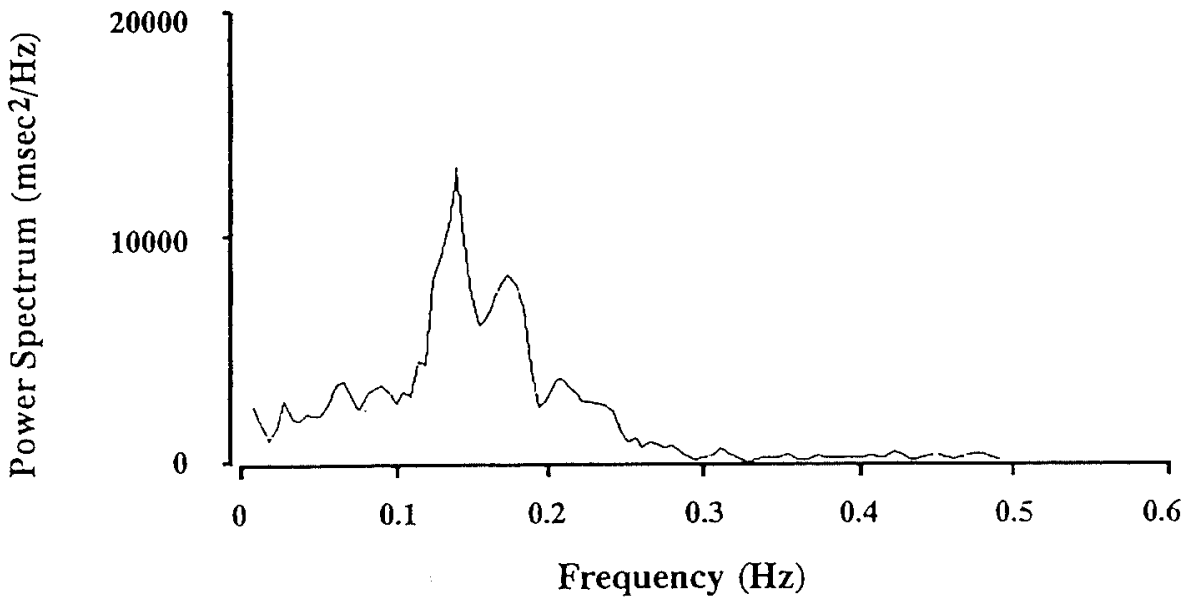

Fig. 2. Representative tachogram (A) and histogram (B) of RR intervals and power spectrum of heart rate variability (C) obtained from about $1 \mathrm{hr}$ ECG record.

respiratory related periodicity in the horse with atrial fibrillation and the predominant parasympathetic activity may modulate the intrinsic behavior of the AV node during atrial fibrillation. 
The ventricular response to atrial fibrillation depends on the rate of atrial impulses arriving at the AV node and on its intrinsic behavior to this input [14]. There is evidence that the autonomic nervous system alters the ventricular response to atrial fibrillation [3]. Furthermore, because atropine administration increased the ventricular rate, vagal tone dominates sympathetic drive during atrial fibrillation [6]. Meijler et al. [11] reported that a considerable drop in blood pressure during the long RR intervals may cause autonomic nervous interference with the AV conduction properties. Our results strongly support these earlier observations, because the predominant parasympathetic nervous function modulates the ventricular rhythms in the horse with atrial fibrillation. However, because the horse in the present case was more than 30 years old, the persistent type of atrial fibrillation might arise from pathological causes. Moreover, we currently have no data about the autonomic nervous functions under a paroxysmal type of atrial fibrillation in the horse. Therefore, further studies will be needed to elucidate whether this result is true for all horses with atrial fibrillation. We believe that the power spectral analysis of heart rate variability provides a very useful approach for these studies.

ACKNOWLEDGMENTS. The authors thank Mr. Koichiro Tanigawa and Mr. Takahide Tanigawa of Tanigawa Ranch for their cooperation in this study. We are also grateful to Dr. Hironori Oki, Dr. Masa-aki Oikawa, Equine Research Institute, Dr. Hitoshi Masuzawa, Hidaka Yearling Training Farm, and Dr. Akio Amada, Blood Horse Training Center for their valuable advice.

\section{REFERENCES}

1. Akselrod, S., Gordon, D., Ubel, F. A., Shannon, D. C., Barger, A. C. and Cohen, R. J. 1981. Science 213: 220-222.

2. Bertone, J. J. and Wingfield, W. E. 1987. Compend. Contin. Educ. Pract. Vet. 9: 763-711.

3. Bleecker, E. T. and Engel, B. T. 1973. Psychosom. Med. 35: 161-175.

4. Deem, D. A. and Fregin, G. F. 1982. J. Am. Vet. Med. Assoc. 180: 261-265.

5. Holmes, J. R., Henigan, M., Williams, R. B. and Witherington, D. H. 1986. Equine Vet. J. 18: 37-42.

6. Horan, L. G. and Kistler, J. C. 1961. Circ. Res. 9: 305-311.

7. Ishii, K., Kuwahara, M., Tsubone, H. and Sugano S. 1996. Lab. Anim. 30: 359-364.

8. Kuwahara, M., Hashimoto, S., Ishii, K., Yagi, Y., Hada, T., Hiraga, A., Kai, M., Kubo, K., Oki, H., Tsubone, H. and Sugano S. 1996. J. Auton. Nerv. Syst. 60: 43-48.

9. Kuwahara, M., Hiraga, A., Nishimura, T., Tsubone, H. and Sugano, S. 1997. J. Equine Sci. 8: 39-42.

10. Kuwahara, M., Yayou, K., Ishii, K., Hashimoto, S., Tsubone, H. and Sugano, S. 1994. J. Electrocardiol. 27: 333-337.

11. Meijler, F. L., Kroneman, J., Van der Tweel, I., Herbschleb, J. N., Heethaar, R. M. and Borst, C. 1984. J. Am. Coll. Cardiol. 4: 316-323.

12. Morris, D. D. and Fregin, G. F. 1982. Cornell Vet. 72: 339349.

13. Pagani, M., Lombardi, F., Guzzetti, S., Rimoldi, O., Furlan, R., Pizzinelli, P., Sandrone, G., Malfatto. G., Dell'Orto, S., Piccaluga, E., Turiel, M., Baselli, G., Cerutti, S. and Malliani, A. 1991. Circ. Res. 59: 178-193.

14. Rawles, J. 1992. p. 153. Atrial Fibrillation. Springer Verlag, London.

15. Reef, V. B. 1985. Vet. Clin. North Am.: Equine Pract. 1: 275-288. 